\title{
Development of the Electromagnetic Calculation Software Package for a Synchronous Motor Based on the Field-Circuit Combination Algorithm
}

\author{
Feng Chen $\mathbb{D}^{1},{ }^{1}$ Qingquan Gan $\mathbb{D}^{2,3}$ and Boyi $\mathrm{Yu}^{3}$ \\ ${ }^{1}$ College of Artificial Intelligence, Zhejiang College of Security Technology, Wenzhou 325016, Zhejiang, China \\ ${ }^{2}$ School of Electrical Engineering, Beijing Jiaotong University, 10044 Beijing, China \\ ${ }^{3}$ School of Mechanical, Electronic, and Control Engineering, Beijing Jiaotong University, 10044 Beijing, China \\ Correspondence should be addressed to Qingquan Gan; 18291226@bjtu.edu.cn
}

Received 3 June 2021; Revised 13 June 2021; Accepted 17 June 2021; Published 10 August 2021

Academic Editor: Jian Su

Copyright (c) 2021 Feng Chen et al.. This is an open access article distributed under the Creative Commons Attribution License, which permits unrestricted use, distribution, and reproduction in any medium, provided the original work is properly cited.

\begin{abstract}
Nowadays, domestic motor manufacturers often use high-speed circuit and magnetic circuit parameters in engine models. In addition, the calculation accuracy is low and cannot fully meet the requirements of a modern engine design. The field-circuit combination algorithm is based on electric field analysis, which avoids many rough assumptions and empirical formulas; its calculations are more accurate and reliable than traditional algorithms. It is used to analyze and calculate the steady-state operating conditions and characteristics of motors, which can effectively improve the design level of China's boss synchronous motors. This article mainly introduces software algorithms and auxiliary FDTD methods. In this article, we use the field-circuit coupling algorithm to develop an electromagnetic calculation software package for synchronous motors and establish a mathematical model for the potential field-circuit coupling algorithm. We use the cassette electromagnetic calculation program to solve the model, evaluate the synchronous motor, and use historical data to modify the model and improve the accuracy of the development-state evaluation of the electromagnetic calculation software package for the synchronous motor based on the motor electromagnetic circuit coupling algorithm. The experimental results of this article show that the field circuit combination algorithm increases the development of the electromagnetic calculation software package for synchronous motors by $55 \%$ and reduces the false alarm rate and false alarm rate. Finally, by comparing the phases of traditional algorithms, we analyzed the excitation circuit combination algorithm to calculate the parameters and performance of the synchronous motor.
\end{abstract}

\section{Introduction}

1.1. Background and Significance. At present, domestic motor manufacturers often use the method of calculating circuit and magnetic circuit lumped parameters and the numerical calculation method of an electromagnetic field when designing motors [1]. From the perspective of practical application, both of them have certain defects $[2,3]$. However, the calculation process of the latter is complicated, which is difficult for technicians to understand [4]. Therefore, neither of these two methods can achieve satisfactory results in practical application [5]. When the magnetic field circuit is integrated into the algorithm to analyze the motor parameters and effi- ciency, the calculation software formation is easy to use and can achieve multigroup and multifeature calculations, so that users can select multiple groups of different forms of calculation data and characteristic curve output [6]. Finally, the software package with an accurate algorithm, simple operation, friendly interface, and suitability for domestic motor designers is jointly developed, which will help to improve the current motor design level in China and improve the competitiveness of motor products [7].

1.2. Related Work. Dusek J studied the influence of the slotpole combination on the interturn short-circuit current of fault-tolerant permanent magnet motors $[8,9]$. The 
multiobjective genetic algorithm is used to establish a twodimensional subdomain field calculation model, and the design and performance prediction of the FT-PM motor are carried out. The electromagnetic loss of the motor, including iron loss, magnet loss, and winding loss, is calculated systematically by using this analysis tool. In the postprocessing stage, the one-dimensional analysis method is adopted for steering fault analysis [5]. This method calculates the self-induction and mutual induction of fault turns and normal turns according to the fault position, so as to calculate the fault current when the fault position [10] is considered. However, there are some unstable factors in the process of the experiment, resulting in inaccurate experimental results.

1.3. Research Innovation Points. The innovation of this article is the use of a modern computing technology based on the field-road combination calculation method. According to the steady-state electromagnetic ideal straight line and the safety threshold range of the synchronous motor, as a basis for judging the process occurring in the motor, a fieldcircuit combination calculation method is established. The probabilistic representation of the steady-state electromagnetic state of the synchronous motor provides a basis for the design and actual operation of the electromagnetic calculation software package of the synchronous motor.

\section{Development Method of the Electromagnetic Calculation Software Package for a Synchronous Motor Based on the Circuit Field Combination Algorithm}

2.1. Software Algorithm. These algorithms are designed according to the idea of modularity. After the function of each module is realized through programming, it will be packaged into functions and open interfaces for calling. Because the control frequency of the speed loop and the current loop changes, the response value is obtained through Clark transformation and Park change. The current loop frequency is high and the speed loop frequency is low. Therefore, calibration is required to set the meter to a fixed value; when the calculated value is equal to $k, m$, perform speed control. When the count value is less than $m$, only the closed loop control of the current is performed. After the control signal is generated, the required tube conduction time can be obtained through the IPM conversion and the space vector calculation module and finally undergo a DSP event. The manager generates the corresponding PWM signal.

2.2. Second-Order FDTD Method. This article chooses the longest wavelength in a specific time period as the target. First of all, since the size change is small compared with the rest of the entire motor, this change will affect the weight and inertia and slightly affect the transportation cost, but this change will affect the electrical conductivity and the impact cannot be ignored. Second, customized technical guidelines for the most expensive car turning lanes depend on driving conditions. Third, the actual gravity and shape of the traction vehicle are very close to a sine wave, which is why the actual fixed gravity is not much different from its value. The high
TABLE 1: The calculation and actual measurement of the original prototype and the comparison of different tooth layer size schemes.

\begin{tabular}{lcccc}
\hline Plan description & $b 3$ & $h 3$ & $g 3$ & $\begin{array}{c}\text { Tmax } \\
(\mathrm{kg} \cdot \mathrm{cm})\end{array}$ \\
\hline Original prototype calculation & 013669 & 015000 & 0.0282 & 6115191 \\
Initial value scheme 1 & 0.4032 & 013871 & 0.0333 & 7513800 \\
Initial value scheme 2 & 013891 & 013871 & 0.033 & 7611068 \\
\hline
\end{tabular}

value of the initial wave can be easily obtained from the static torque value, and the average static torque value can be obtained by changing the magnetic force and the rotating side. The magnetic coenergy change of the tooth moment angle $\theta$ from 0 to $\pi$ is

$$
\Delta E=E_{\pi}-E_{0}
$$

where $E_{\pi}$ and $E_{0}$ are the total magnetic coenergy of the motor when the corresponding tooth pitch angles is $\pi$ and 0 , respectively. The increment of the tooth moment angle is expressed in the mechanical angle as

$$
\Delta \theta=\frac{\pi}{F_{R}}
$$

In this article, the mathematical field combination circuit model combines their respective field and circuit advantages. The characteristic of this method is to solve the two-way electromagnetic field problem. For the magnetic field problem of the tooth layer unit in the tooth layer range, for the size and material of the normal tooth range layer, the corresponding layer first calculates the magnetic permeability and specific magnetic energy data and then calculates the magnetic field of the tooth layer unit and other parts of the motor. The conductivity forms a nonlinear magnetic network, which can better reflect the electromagnetic process of the motor, and the characteristics of the motor can be calculated by analyzing the magnetic network.

\section{Development Experiment of the Electromagnetic Calculation Software Package for the Synchronous Motor Based on the Field-Circuit Combination Algorithm}

3.1. Development of the Electromagnetic Calculation Software Package for the Synchronous Motor Based on the Field-Circuit Combination Algorithm. In this paper, when one phase is energized and the winding current is $551 \mathrm{~A}$ and the initial values of the two sets of tooth layer sizes are selected to optimize the design. The calculation results are shown in Table 1. The initial values are $b 3=0.245$ and $h 3=0.148$ for the first time. The maximum static torque of the advantage solution obtained is $7518848 \mathrm{~kg} \cdot \mathrm{cm}$, the initial value of the second time is $b 3=0.138, h 3=0.15$, the maximum static torque of the advantage solution obtained is $7943068 \mathrm{~kg}-\mathrm{cm}$, and the advantage solution is better than the maximum static torque of the original prototype which 


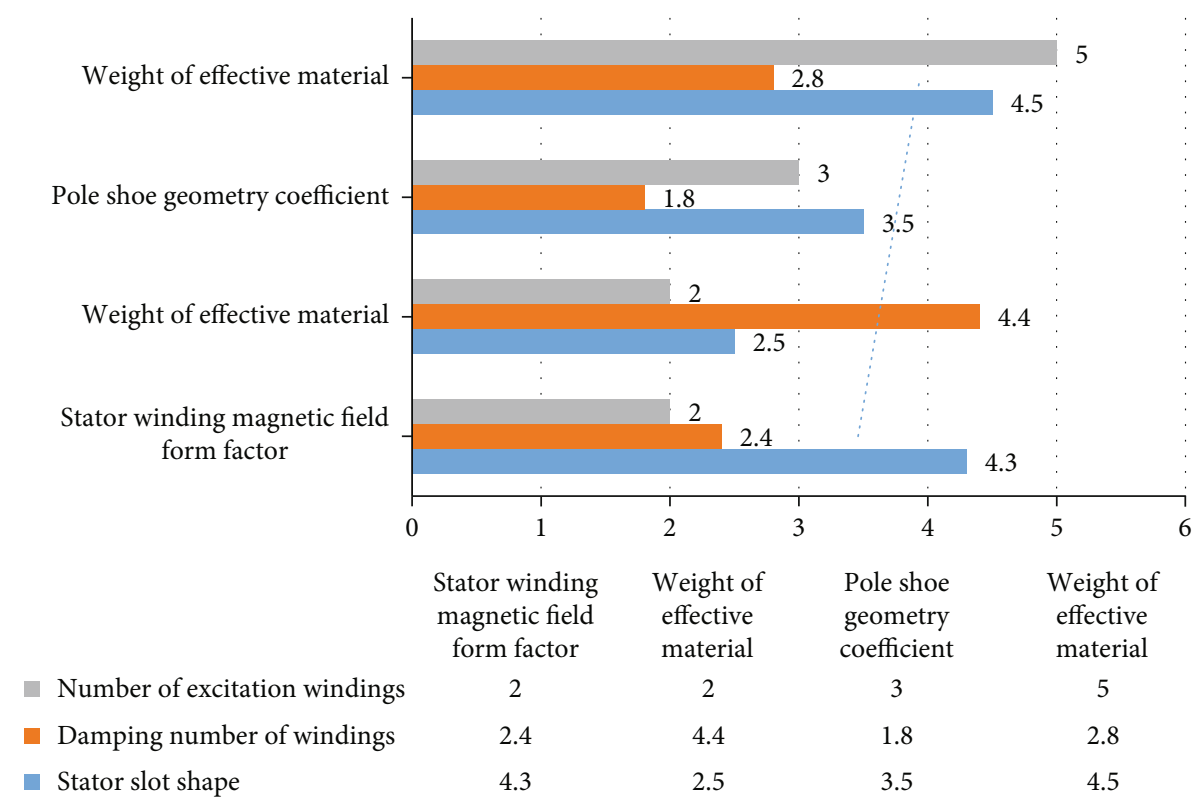

Figure 1: Modular data diagram of control system software.

is large. The comparison between the calculation and the measurement and the comparison with the optimization scheme are shown in Table 1.

As can be seen in Table 1, the measured value of static torque is greater than the calculated value. This is because the calculated value is the maximum value of the fundamental wave and the measured value is the actual maximum value. The relative error between calculation and measurement is $414 \%$, which meets the engineering accuracy requirements.

\section{Modular Design of Control System Software}

The traditional flowchart software program is difficult to read, the algorithm is not easy to change, and the portability is poor. Modular software programs can reduce program complexity, can make program design convenient, can clear logical relationships, are convenient when debugging, and are easy to maintain. Each module program performs a specific subtask and combines all modules according to the system modules to perform the tasks required by the entire system. The software modular design based on the field circuit combination algorithm involves two aspects. First is the modular design of the functions experienced by the software module control, and the second is the field circuit hybrid algorithm design. The task software module is a digital control system. According to the software requirements, all the software can be divided into assembly language to modulate and write the system startup, torque control, power stop control, and serial communication programs. The synchronous motor rotation control program calls the subroutines of the sine and cosine values of the rotor feedback angle to obtain the calculated torque winding current value and calls the inverter. The modular data of the control system software is shown in Figure 1.
It can be clearly seen from the form and can be changed, the inverse transformation subroutine is called, the inverse winding subroutine is not called during rotation, and it is predicted that there is a calculation element feedback by the rotor. Resources are managed by controlling interrupts, that is, before using these resources, the relevant interrupts must be first disabled, and then, the corresponding interrupts after the end of use must be enabled. This kind of management mechanism realized by the corresponding control will cause some delay in the realization of the interrupt subroutine service, but this delay can be reduced by designing a reasonable program structure and enabling the corresponding interrupt. Test results show that this management mechanism is feasible and can control the delay time in the engine cycle to ensure the highest execution efficiency of the program.

\section{Data Analysis of the Development of a Synchronous Motor Electromagnetic Calculation Software Package Based on the Field-Circuit Combination Algorithm}

5.1. Calculation and Actual Measurement of the Original Prototype and the Comparative Analysis of Different Tooth Layer Size Schemes. In the main text, the displacement adjustment values $E$ and $F$ of the motor rotor are set. The error between the values provided by the feedback loop is a vector used to control the engine shutdown radio in the closed loop. Changing the calculation method of the mobile PT rotor radio in the asm file can effectively stop the rotor from stopping. The radial displacement of the rotor moves nearby. In order to make the motor have better speed and suspension performance during operation, the calculation parameters of the EP speed loop and the radial displacement of the rotor $\mathrm{PH}$ must be continuously 


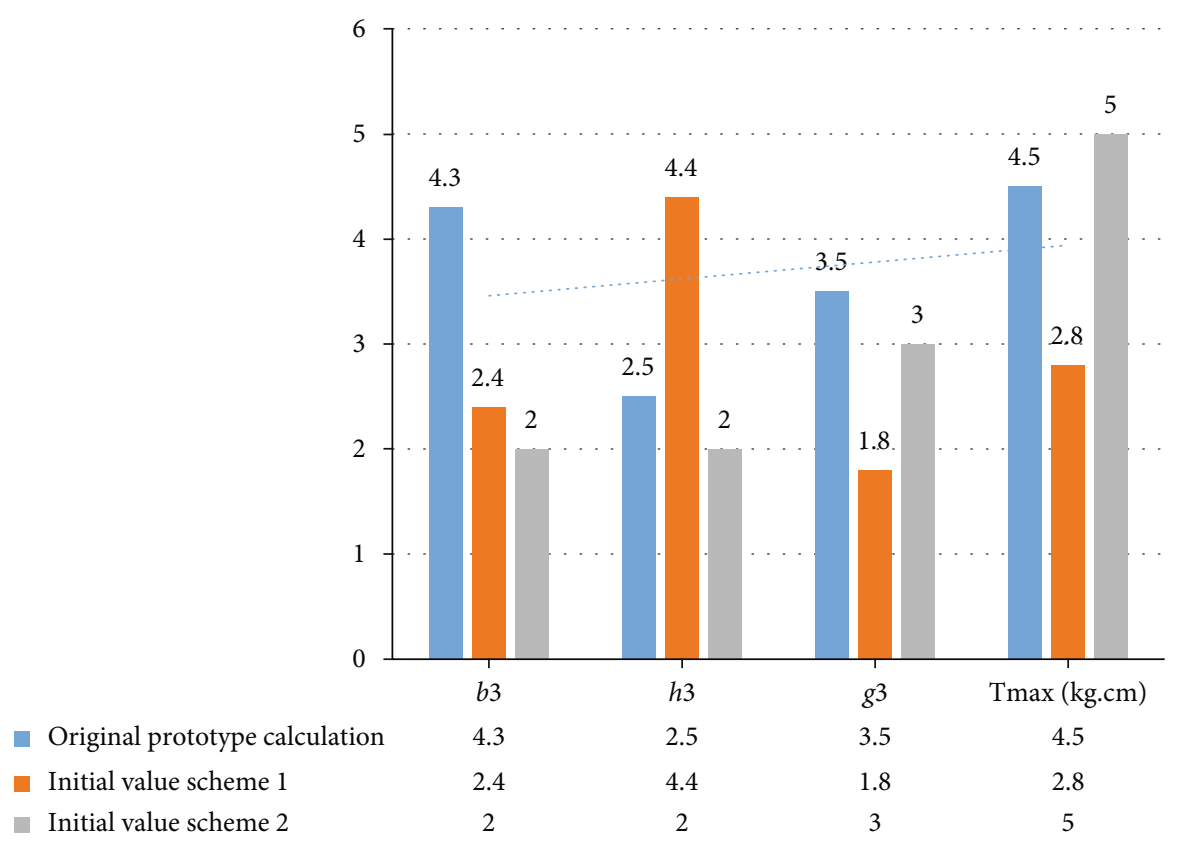

Figure 2: The calculation and actual measurement of the original prototype and the comparison and analysis of different tooth layer size schemes.

TABLE 2: Comparison of software design values and measured values.

\begin{tabular}{lcccc}
\hline Project & Effectiveness (\%) & Power factor & Starting current multiple & Starting torque multiple \\
\hline Design value & 99.25 & 0.894 & 6.58 & 2.067 \\
Measured value & 84.56 & 0.994 & 7.54 & 2.088 \\
Deviation & 78.94 & 0.794 & 9.28 & 0.027 \\
\hline
\end{tabular}

adjusted, but each time the calculation parameters are adjusted, the motor must stop and the system must be a compiled software program. Accurately judge the running performance of the engine and increase the complexity of the test. The research team has developed a basic home computer interface for the BP management system. The results of the bearingless motor test and alignment using this human-computer interaction strategy show that the test and alignment are convenient and simple, the connection is stable and reliable, and the motor suspension has good torque and performance. The model is the original calculation and actual measurement of the machine, and the results of the comparison and analysis of the different size schemes of the tooth layer are shown in Figure 2.

According to the calculation of the actual prototype, the actual measurement, and the comparison of different tooth layer size schemes, the groups are first sorted in proportion, and then, several high-frequency feature words are selected for analysis in each group. The last topic is to get the words, relationships, and meanings of the attributes. This result proves the accuracy of the actual type of calculation and actual measurement, as well as the comparative analysis of various sizes of tooth size schemes. Not only that, even if the proportion is as low as $54 \%$, the results are still satisfactory. It can be seen from the statistical data that the two experimental results show that under different conditions, test plan 1 is better than test plan 2 .

It should be noted that as the level of use increases, the efficiency of the system continues to improve. Especially when the completion rate reaches $7 \%$, the performance improvement of the system is very obvious, the performance improvement of the system is very close, and the subsequent completion rate and the result of the system performance improvement are usually the same.

\subsection{Function Analysis of Electromagnetic Calculation} Software for the Synchronous Motor. Synchronous motor electromagnetic calculation software is mainly used for electromagnetic calculation of multiphase asynchronous motors. The representative values in Table 1 are the results calculated by the software in the table, and the measured values are the actual measurement results under the same parameters. As shown in Table 2.

By analyzing the data in Table 2, it can be determined that the design parameters of each parameter are the same as the estimated value and the purpose of writing the programming software is to quickly and accurately obtain the results, so the results indicate that the mathematical software is running and can be used. After the calculation is completed, when you click the "Exit and Save" button, the 


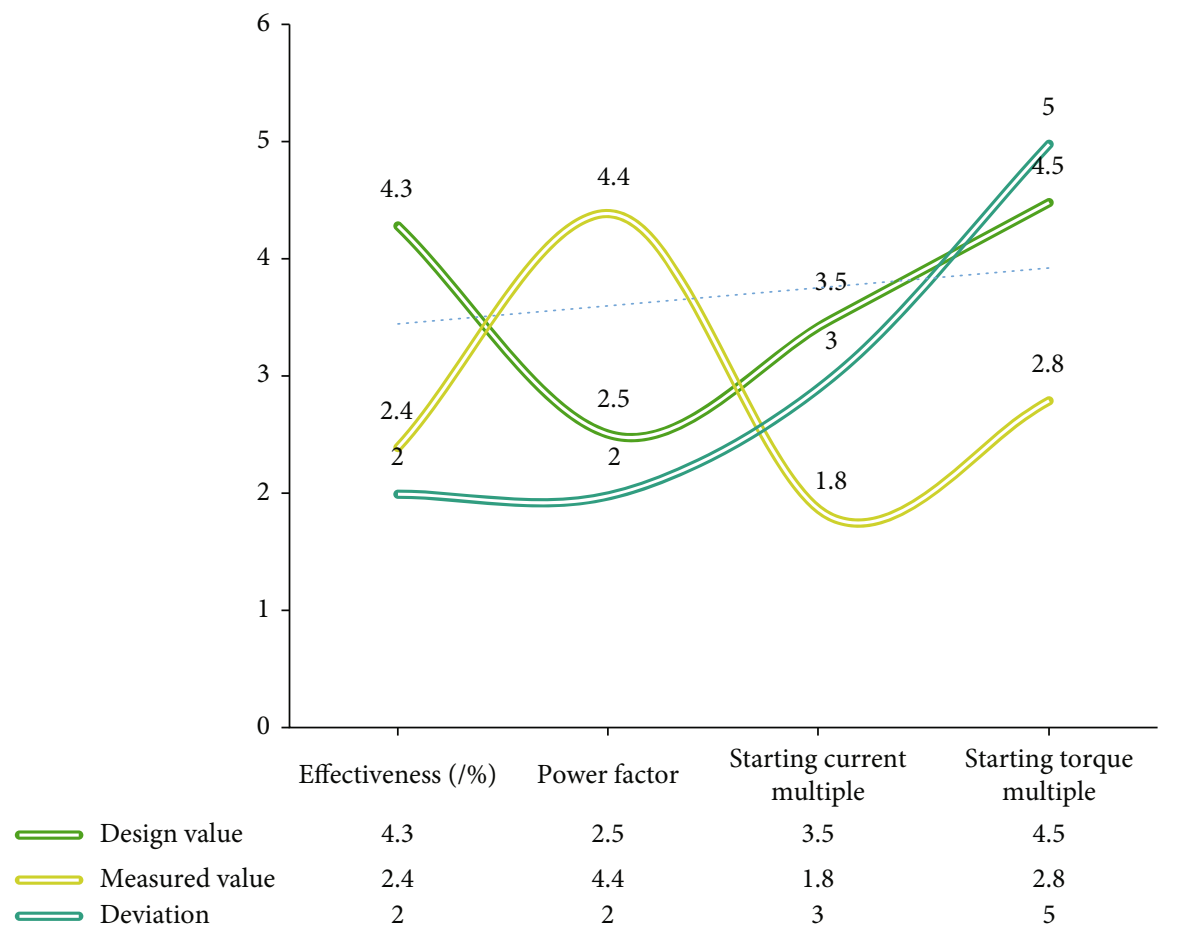

FIgURE 3: Data analysis of software design values and measured values.

readable result will be displayed in the context below, so you can choose a wise solution based on the result. It can be seen that using electrical calculator software for multiple power levels can reduce the design time and improve performance. The data analysis of software design value and estimated value is shown in Figure 3.

\section{Conclusions}

According to the characteristics of the system and the need to complete the task, the overall design of the task. The scheme selection of the entire operating system is carried out, and the parallel control research and design are carried out from the master slave control and parallel control. Based on the determination of the scheme, this paper carried out the hardware circuit design of the system and the interface circuit design of the bus and wrote the main model, as well as the specific operation and model data.

In this paper, some achievements have been made on the research model of the electromagnetic calculation software package for synchronous motors and there are still some shortcomings on the basis of the field circuit combination algorithm. There are still many methods for in-depth research in the development and research of the electromagnetic calculation software package for synchronous motors based on the combined field circuit algorithm. Due to space and personal skills, there are many steps in the decisionmaking process that have not been involved. In addition, from the perspective of theory and simulation, the actual application effect of the improved algorithm can only be compared with traditional models.

\section{Data Availability}

The data underlying the results presented in the study are available within the manuscript.

\section{Conflicts of Interest}

The authors declare that they have no conflicts of interest.

\section{Authors' Contributions}

Feng Chen is the experimental designer and the executive of the experimental research of this research. Qingquan Gan completed the data analysis and the writing of the first draft of the paper. Boyi $\mathrm{Yu}$ is the executive of the experimental research of this research and participated in the writing and revision. Feng Chen and Qingquan Gan are co-first authors.

\section{References}

[1] H. Zhao, X. Chen, and J. Cheng, "Virtual instrument based fuzzy control system for PMLSM drive," Research Output of Integration Institute, vol. 22, no. 1, pp. 299-303, 2019.

[2] S. Yongxian and Z. Xianjin, "Calculation and simulation of electromagnetic wave propagation path loss based on MATLAB," International Journal on Smart Sensing \& Intelligent Systems, vol. 9, no. 4, pp. 1943-1970, 2016.

[3] W. Li, L. Li, H. Gao, D. Li, X. Zhang, and Y. Fan, "Influence of direct-connected inverter with one power switch open circuit fault on electromagnetic field and temperature field of permanent magnet synchronous motor," IET Electric Power Applications, vol. 12, no. 6, pp. 815-825, 2018. 
[4] W. Hu, X. Zhang, H. Yin, H. Geng, Y. Zhang, and L. Shi, "Analysis of magnetic field and electromagnetic performance of a new hybrid excitation synchronous motor with dual-V type magnets," Energies, vol. 13, no. 6, p. 1501, 2020.

[5] J. Xu, X. Yang, W. Li, J. Zheng, Y. Wang, and M. Fan, "Research on semi-active vibration isolation system based on electromagnetic spring," Mechanics and Industry, vol. 21, no. 1, p. 101, 2020.

[6] A. Mahapatro and P. M. Khilar, "Fault diagnosis in wireless sensor networks: a survey," IEEE Communications Surveys \& Tutorials, vol. 15, no. 4, pp. 2000-2026, 2013.

[7] H. Qiu, X. Zhao, C. Yang, R. Yi, and Y. Wei, "Influence of open-circuit fault on electromagnetic field of high-speed permanent magnet generator with Gramme ring windings," IET Electric Power Applications, vol. 14, no. 2, pp. 220-225, 2020.

[8] L. Li, J. Zhang, C. Zhang, and J. Yu, "Research on electromagnetic and thermal issue of high-efficiency and high-powerdensity outer-rotor motor," IEEE Transactions on Applied Superconductivity, vol. 26, no. 4, pp. 1-5, 2016.

[9] J. H. Kim, C. J. Hyeon, S. H. Chae et al., "Design and analysis of cooling structure on advanced air-core stator for megawattclass HTS synchronous motor," IEEE Transactions on Applied Superconductivity, vol. 27, no. 4, pp. 1-7, 2017.

[10] P. Todoroff and P. Langellier, "Comparison of empirical and partly deterministic methods of time domain reflectometry calibration, based on a study of two tropical soils," Soil and Tillage Research, vol. 45, no. 3-4, pp. 325-340, 1998.

[11] F. Lin, S. Zuo, and X. Wu, "Electromagnetic vibration and noise analysis of permanent magnet synchronous motor with different slot-pole combinations," IET Electric Power Applications, vol. 10, no. 9, pp. 900-908, 2016. 